University of Nebraska - Lincoln

DigitalCommons@University of Nebraska - Lincoln

$1-2012$

\title{
A novel fixed-target task to determine articulatory speed constraints in persons with amyotrophic lateral sclerosis
}

\author{
Antje Mefferd \\ Wichita State University, antje.mefferd@wichita.edu \\ Jordan R. Green \\ University of Nebraska-Lincoln, jgreen4@unl.edu \\ Gary L. Pattee \\ University of Nebraska Medical Center-Omaha
}

Follow this and additional works at: https://digitalcommons.unl.edu/specedfacpub

Part of the Special Education and Teaching Commons

Mefferd, Antje; Green, Jordan R.; and Pattee, Gary L., "A novel fixed-target task to determine articulatory speed constraints in persons with amyotrophic lateral sclerosis" (2012). Special Education and Communication Disorders Faculty Publications. 70.

https://digitalcommons.unl.edu/specedfacpub/70

This Article is brought to you for free and open access by the Department of Special Education and Communication Disorders at DigitalCommons@University of Nebraska - Lincoln. It has been accepted for inclusion in Special Education and Communication Disorders Faculty Publications by an authorized administrator of DigitalCommons@University of Nebraska - Lincoln. 
Published in Journal of Communication Disorders 45:1 (January-February 2012), pp. 35-45;

doi: 10.1016/j.jcomdis.2011.09.002

Copyright (c) 2011 Elsevier Inc. Used by permission.

Submitted May 21, 2010; revised September 6, 2011; accepted September 19, 2011; published online September 26, 2011.

\title{
A novel fixed-target task to determine articulatory speed constraints in persons with amyotrophic lateral sclerosis
}

\author{
Antje S. Mefferd, ${ }^{1}$ Jordan R. Green, ${ }^{1,2}$ and Gary Pattee ${ }^{2}$ \\ 1. Department of Special Education and Communication Disorders, University of Nebraska-Lincoln, Lincoln, NE \\ 2. Munroe-Meyer Institute, University of Nebraska Medical Center, Omaha, NE \\ Corresponding author - A. S. Mefferd, Department of Communication Sciences and Disorders, \\ College of Health Professions, Wichita State University, 401 E Ahlberg Hall, Wichita, KS 6726o-o075, USA; \\ tel 316 978-7345, fax 316 978-3291, email antje.mefferd@wichita.edu
}

\begin{abstract}
Purpose: The goal of this study was to determine if talkers with ALS are limited in their ability to increase lower lip and jaw speed at an early stage of the disease when their speaking rate and intelligibility are only minimally or not affected.

Method: A novel metronome paced fixed-target task was used to assess movement speed capacities during lower lip and jaw oscillations in seven talkers with ALS and seven age and gender matched controls.

Results: Lower lip peak speeds were significantly lower in talkers with mild ALS than in healthy talkers suggesting a lower lip speed constraint in talkers with mild ALS. Jaw peak speeds tended to be lower, but jaw displacements tended to be larger in talkers with mild ALS than in healthy talkers. Because greater speeds are typically expected for larger displacements, outcomes also suggest a jaw speed constraint in talkers with mild ALS.

Conclusions: Lower lip and jaw peak speeds may be sensitive measures to identify bulbar motor performance decline at an early stage of the disease when speaking rate and intelligibility are only minimally affected.

Learning Outcomes: The reader will be able to explain two different articulatory strategies to increase speaking rate and understand why fast speech tasks and diadochokinetic pseudo-speech tasks are not suited to assess articulatory speed capacity in healthy and impaired talkers. The reader will also be able to explain how orofacial movement speed capacity can be tested using a fixed-target task and how ALS affects lower lip and jaw speed capacities during the early stages of the disease.
\end{abstract}

Keywords: motoneuron degeneration, ALS, kinematic analysis, movement speed, speaking rate

\section{Introduction}

Amyotrophic lateral sclerosis (ALS) is a progressive neuromuscular disease that affects the upper and lower motoneurons resulting in progressive weakness and fatigue of lip, tongue, and jaw muscles (Brooks, 1996; DePaul and Brooks, 1993; DePaul et al., 1988; Langmore and Lehman, 1994). Although slowing of speaking rate and deterioration in speech intelligibility have been well documented with disease progression (i.e., Ball, Beukelman, \& Pattee, 2002), little is currently known about underlying declines in articulatory performance. Recent findings suggest that declines of orofacial control and coordination may predate overt changes in speaking rate and intelligibility (Yunusova et al., 2010). A better understanding of motor performance decline in the bulbar system is, therefore, warranted and may expedite the diagnosis, provide objective outcome measures for therapeutic drug trials, and allow 
predictions about the rate of speech decline within an individual. This later clinical goal is essential for facilitating and optimizing the transition to non-speech communication modes (Beukelman et al., 2011; Yorkston et al., 1993).

Although slowed articulation is a common perceptual feature of ALS (Darley et al., 1969a, 1969b), only a few investigations have examined articulatory speed (Hirose et al., 1982a, 1982b; Yunusova et al., 2008, 2010). These studies have shown that, relative to healthy controls, individuals with ALS exhibited slower articulatory speeds during diadochokinetic tasks (Hirose et al., 1982a, 1982b) and word productions (Yunusova et al., 2008). Because participants in these studies showed already a perceptible decline in speech intelligibility, it is currently unknown if articulatory speed is affected during the early stages of the disease when speaking rate and intelligibility are not or only minimally affected. If slowed oral movements are observed during the early stages of the disease, then articulatory speed may be a sensitive measure to capture incremental motor performance declines of the bulbar system in talkers with ALS. In addition, declines in articulator speed may also signal impending declines in speaking rate and intelligibility in these talkers.

One limitation of prior studies examining articulatory speed was that the articulatory strategy was not controlled for. It is commonly known that most talkers economize their efforts when speaking (i.e., Lindblom, 1990; Perkell et al., 1997). For example, when speaking fast many talkers reduce their displacements (Kuehn and Moll, 1976; Mefferd and Green, 2010). This articulatory strategy allows talkers to shorten movement durations without actually increasing articulatory speed. Therefore, diadochokinetic pseudo-speech tasks and fast speech tasks are not suitable to assess a talker's speed capacity; the ability to increase speed can only be examined in a task that limits the talker from reducing articulatory displacements when speaking faster. In the limb literature, fixed-target tasks have been used to assess the speed capacity of arm movements while controlling movement displacement, for example during reaching-to-grasp or reaching-to-point tasks (i.e., Kelly and Bastian, 2005; Milner and Ijaz, 1990; Rand et al., 2006). To our knowledge, a similar approach has not been used to investigate orofacial motor performance.

This study aimed to reveal potential orofacial speed constraints in talkers with ALS during the early stages of the disease when speaking rate and intelligibility are not or only minimally affected. As a first step, lower lip and jaw speed capacities were tested using a novel metronome paced fixed-target task approach. In the future, the assessment of tongue speed with experimental control over articulatory displacements is also warranted to obtain a more complete understanding of orofacial speed capacities in talkers with ALS.

In this study, we tested the hypothesis that talkers with mild ALS would not be able to increase their lower lip and jaw speed to the same extent as healthy talkers under a metronome paced fixed-target task condition. In addition, we hypothesized that during the fixed-target tasks, talkers with mild ALS would not be as accurate in their ability to match fast metronome paces as healthy talkers and that accuracy would decrease with increase in metronome pace. Two talkers with severe ALS were also included in this study. Although these two participants are likely to be constrained in their ability to increase movement speeds and accurately match fast metronome paces under a fixed-target condition, their potential range of movement speed and duration modulation was explored.

\section{Methods}

\subsection{Participants}

Seven talkers with ALS and seven age and gender matched controls were included in this study. One talker with ALS and one control were excluded. The talker with ALS had difficulty with uncontrollable laughing and crying (pseudobulbar affect), which interfered with the speech tasks. The age and gender-matched control person was excluded because the contour of her chin did not permit proper placement of the strike target during the jaw fixedtarget task (see also Section 4.5).

Talkers with ALS had been diagnosed by a certified neurologist. All participants passed a hearing screening at $0.5,1,2$, and $4 \mathrm{kHz}$ at $30 \mathrm{~dB}$ and had no self-reported history of neurological diseases other than ALS. Healthy talkers were between 48 and 68 years of age $(M=55.4)$. Talkers with ALS were between 48 and 74 years of age $(M=56.6)$. Further demographics and speech characteristics of talkers with ALS can be found in Table 1. Standard English was their first and only language. Except for talkers ALS 3 and ALS7, all participants with ALS reported taking pramipexole, a dopamine agonist that is currently being tested in several clinical trials.

Table 1. Demographics and speech characteristics of talkers with ALS.

\begin{tabular}{lllllll}
\hline ID & Gender & Age & Initial type of ALS & SIT & Severity & Speech characteristics \\
\hline ALS1 & F & 74 & Spinal & 100 & Mild & Vocal fatigue \\
ALS2 & M & 52 & Spinal & 99 & Mild & Mild articulatory imprecision, mild vocal hoarseness \\
ALS3 & F & 53 & Bulbar & 31 & Severe & Hypernasality, severe articulatory imprecision \\
ALS & M & 55 & Spinal & 98 & Mild & Mild articulatory imprecision \\
ALS5 & F & 50 & Bulbar & 25 & Severe & Hypernasality, severe articulatory imprecision, low pitch \\
ALS7 & F & 48 & Bulbar & 100 & Mild & Mild articulatory imprecision \\
ALS8 & F & 64 & Spinal & 100 & Mild & Mild articulatory imprecision low pitch, mild vocal hoarseness \\
\hline
\end{tabular}



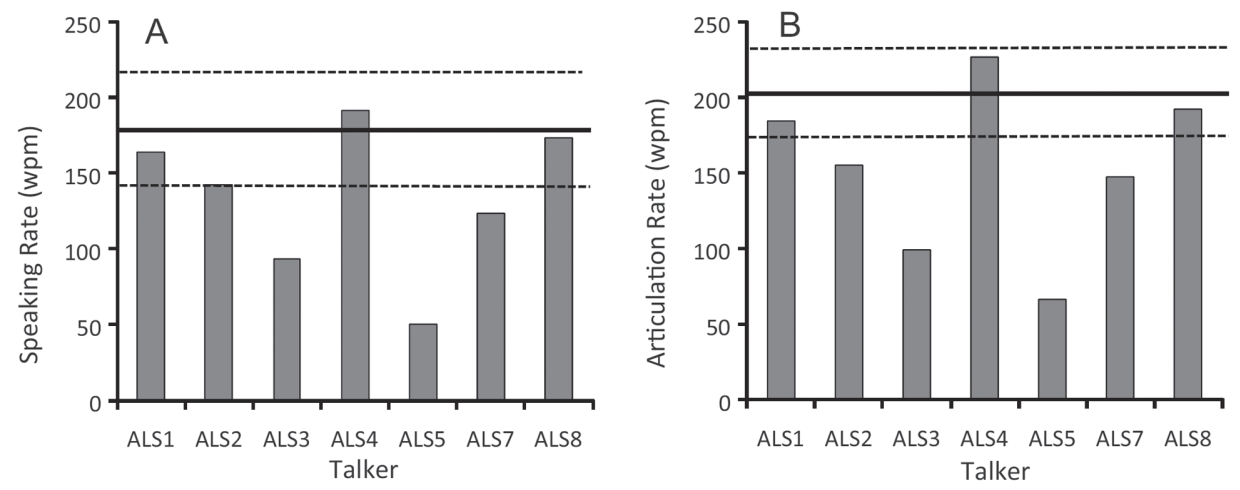

Figure 1. Individual speaking rates (A) and articulatory rates (B) of each talker with ALS. The mean of controls is marked in each panel by a black solid line. The dashed lines represent one standard deviation above and below the control group's mean.

The speech intelligibility and rate of talkers with ALS and healthy controls was measured using the Sentence Intelligibility Test (SIT; Yorkston, Beukelman, Hakel, \& Dorsey, 2007). SIT scores are displayed in Table 1. The severity ratings and speech characteristics, which were judged by a certified speech-language pathologist, are also provided. Speaking rates were calculated using sentence durations that included all pauses within each sentence; thus, the longer the pause times during each sentence, the lower the number of words spoken per minute. Articulatory rates were calculated using sentence durations that disregarded pauses of $200 \mathrm{~ms}$ or longer. Consequently, the measure of articulatory rate is, in contrast to speaking rate unaffected by pauses. Figure 1 displays speaking rate (panel A) and articulatory rate (panel B) of each talker with ALS. Additionally, in each panel, the mean of all control talkers is marked by a black solid line and the dashed lines represent one standard deviation of the control group's mean.

\subsection{Instrumentation}

\subsubsection{Motion analysis system}

Movements of the lower lip and jaw were captured using a three-dimensional optical motion capture system (Motion Analysis, Ltd.) consisting of eight Eagle cameras with a resolution of 1.3 million pixels at $1280 \times 1024$ full resolution. Figure 2 shows the placement of the reflective markers. Seven small $(\sim 2 \mathrm{~mm})$ reflective spheres were placed on each participant's jaw and mouth. Eight reflective reference markers were placed on the nose bridge (nasion) and forehead. The movements were sampled at $120 \mathrm{~Hz}$. A miniature microphone was placed on the forehead to record the audio signal. The audio signal was sampled at $44.1 \mathrm{kHz}, 16 \mathrm{bit}$. A digital video was also recorded as a reference during the analysis of movement data.

\subsubsection{Fixed-target device}

Each participant wore a lightweight head mounted device that supported an adjustable rigid bar (the strike plate) that was located underneath the jaw or lower lip (Figure 2). A contact microphone, with an attached lever arm extension, was mounted on a strike plate below the jaw and lip. A small, plastic hemisphere $(\sim 2 \mathrm{~mm})$, which was attached to the end of the lever arm, served as the strike target and provided tactile feedback when it was reached by the articulator. The contact microphone/lever arm apparatus generated a signal spike every time the jaw or lower lip touched the target during the opening position. The contact microphone signal was amplified, digitized, and displayed on a computer monitor in real time during the experiment (Biopac, Biopac Systems, Inc.). This signal provided online verification that the participant touched the strike target with their jaw or lip during the metronome paced oscillations.

\subsubsection{Metronome}

A metronome software program ( http://www.pinkandaint.com/weirdmet.shtml ) was used to elicit the metronome paced lower lip or jaw movements at $1.4 \mathrm{~Hz}$ (slow), $3.3 \mathrm{~Hz}$ (moderate), $5 \mathrm{~Hz}$ (fast), and $6.3 \mathrm{~Hz}$ (very fast). These metronome paces were selected based on repetition rates used in previous studies (Hertrich and Ackermann, 200o; Kuehn and Moll, 1976). The $3.3 \mathrm{~Hz}$ repetition rate approximated the speaking rate of a healthy talker's habitual speech, whereas the $6.3 \mathrm{~Hz}$ pace slightly exceeded the reported repetition rates of healthy talkers during maximum performance tasks. The fastest metronome pace was set purposefully high to avoid ceiling effects in motor performance. The metronome signal was played back via a speaker that was placed approximately two feet away from the head of the participants. The metronome signal was adjusted to a comfortable hearing level.

\subsection{Tasks}

Participants completed two tasks: a jaw fixed-target task and a lower lip fixed-target task. In each task, participants were instructed to whisper the syllable "fuh" at $1.4 \mathrm{~Hz}$ (slow), $3.3 \mathrm{~Hz}$ (moderate), $5 \mathrm{~Hz}$ (fast), and $6.3 \mathrm{~Hz}$ (very fast) 


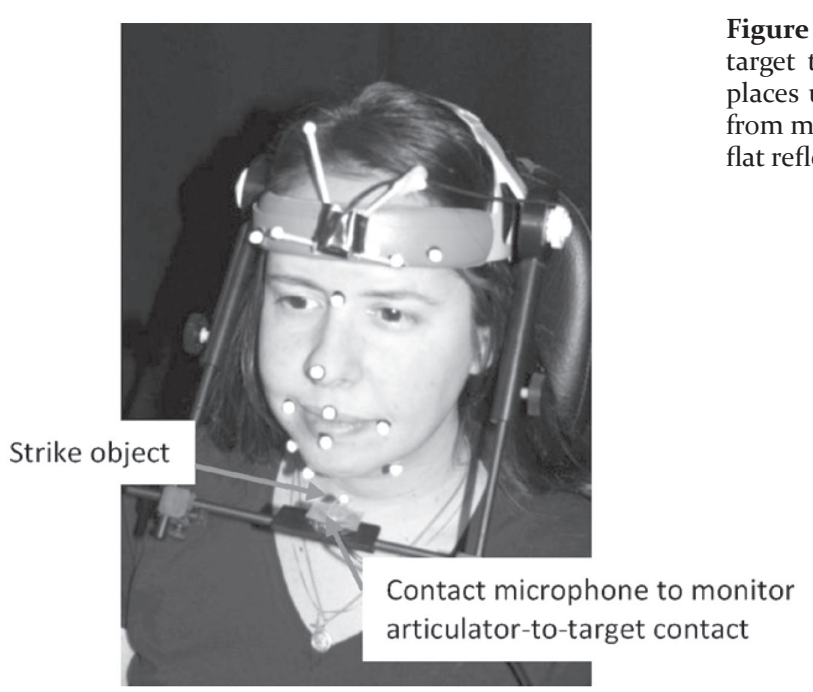

Figure 2. Marker placement and experimental set-up during the jaw fixedtarget task. During the lower lip fixed-target task, the strike object was places underneath the lower lip, a bite block was used to prevent the jaw rom moving, and the small reflective sphere was replaced by a similar sized flat reflective marker.

and strike the target with each whispered repetition. The distance between the articulator and strike target was adjusted for each participant individually by asking the participant to oscillate the articulator according to the slowest metronome pace. The strike target was set to the height where the articulator could reach the target object comfortably and also match the metronome pace. After the slowest metronome pace, no more adjustments were made to the position of the strike target. Strike target contact was monitored continuously throughout the experiment. During data collection, one experimenter observed the signal of the contact microphone on the computer monitor, while another experimenter directly observed the task performance and encouraged the participant to reach the strike target during the fast metronome paces. When the participants did not reach the target, they were reminded of the goals of the task - to touch the target and match the metronome pace. In these cases, the task was repeated up to two additional trials and encouragement was provided before the next metronome pace was recorded. All participants completed all of the metronome paces regardless of how well they were able to match the metronome pace or hit the strike target. The repetitions were produced as long as their breath lasted or until the researcher interrupted the productions because sufficient data were recorded. To isolate the lower lip and establish an inter-incisor distance of $6 \mathrm{~mm}$, a bite block was made for each participant. The bite block was worn during the lower lip fixed-target task.

The syllable "fuh" was used for both fixed-target tasks because the upper central incisors provided a hard upper boundary for the lower lip and jaw movement oscillations during oral closure. The lower boundary during vowel opening strokes was fixed by a rigid object, which was placed under the jaw. The syllable "buh", which is more commonly used to assess lip and jaw movements, was an unsuitable target utterance for the fixed-target tasks because participants were able to move their upper lip closer to their lower lip to achieve bilabial closure as the metronome paces increased. This upper lip compensation was incompatible with the goal of the fixed-target tasks, which was to hold the movement distance of the articulator relatively constant. Participants were asked to whisper "fuh" because it provided auditory feedback, which participants needed to ensure that they realized the acoustic target, but it did not interfere with the contact microphone system that was used to detect when the articulator reached the spatial target during vowel opening.

\subsection{Data analysis}

A signal representing the $3 \mathrm{D}$ Euclidian distance between the right jaw marker and right top head marker was used to represent continuous jaw movements during the jaw fixed-target tasks. The right jaw marker was used because the measurement error due to flesh movement is known to be smaller for flesh-point markers placed on lateral chin regions than on central chin region (Green, Wilson, Wang, \& Moore, 2007). The time history of the 3D Euclidian distance between the center lower lip marker and the right top head marker was used to represent lower lip movements during the lower lip fixed-target task. Using a custom Matlab algorithm, both distance signals were smoothed using an $18 \mathrm{~Hz}$ low pass filter.

Although the fixed-target task was used to maximize the likelihood that a "speed strategy" was implemented to shorten movement durations, some participants had difficulty refraining from reducing displacements, particularly for the lower lip, with increased metronome paces. Therefore, ten oscillations were selected that contained repetitions with the largest articulatory displacements during each trial. The rationale for this approach was that these oscillations best represented repetitions where the strike target was reached or the best effort to reach the target was captured (Figure 3). Thus, for each participant 40 values (10 repetitions $\times 4$ metronome paces) were obtained. In five participants (two talkers with ALS and three healthy talkers), however, only 37-39 eligible oscillations were 


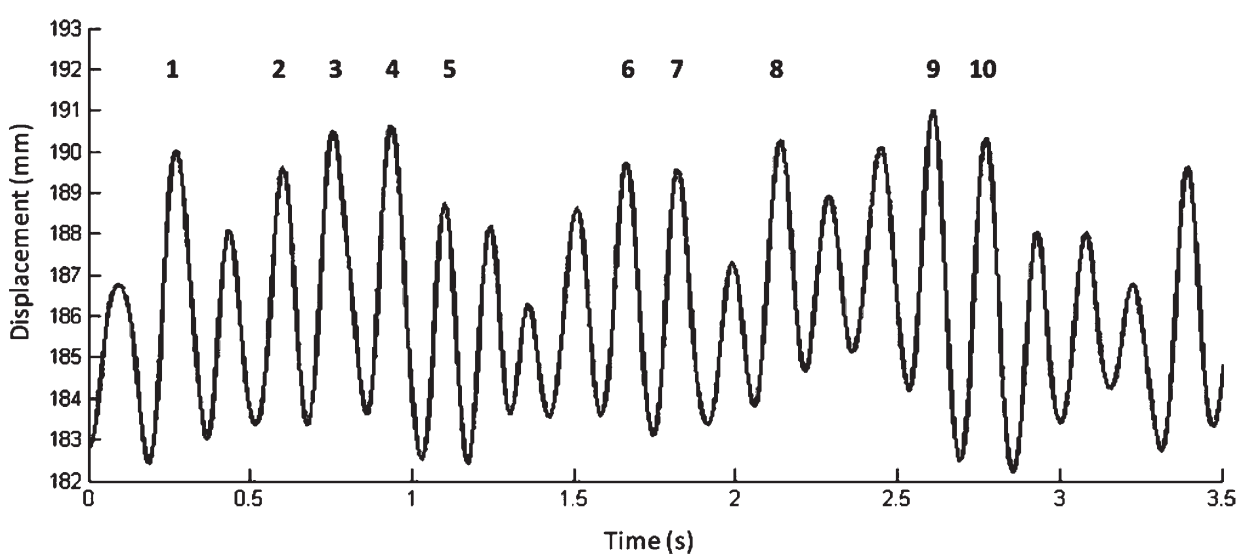

Figure 3. Time history of the 3D Euclidian distance between the right top head marker and right jaw marker. Only the ten largest displacements were selected for data analysis during the jaw and lower lip fixed-target tasks.

obtained. In addition, for one healthy talker, only 29 values were obtained (slow $=10$, moderate $=7$, fast $=3$, very fast $=9$ ) due to tracking errors, especially during the fast pace.

2.4.1. Peak speed and maximum displacement

Peak speeds and maximum displacements of the closing strokes were calculated for each participant using a customized Matlab algorithm. Values of the ten selected lower lip or jaw movement cycles were averaged to obtain one mean value for each participant for each metronome pace. The beginning and ending of each closing stroke was defined by the displacement maximum and minimum of the $3 \mathrm{D}$ Euclidian distance signal of each articulator and the right head marker. The closing stroke was selected because pilot data showed more consistent rate-dependent changes in movement duration and peak speed during the closing strokes than during the opening strokes. This observation is consistent with findings by Kuehn and Moll (1976), who reported larger speed changes for closing strokes than opening strokes when speaking rate was increased.

\subsubsection{Task performance}

To determine each participant's accuracy at matching the metronome pace, the movement durations of each oscillation and syllable repetition were measured using a customized Matlab algorithm. Displacement minimums of the $3 \mathrm{D}$ distance signals indicated the beginning and end of each oscillation. The performance accuracy was determined by calculating the difference between the observed movement duration of each oscillation for a specific metronome pace and the expected movement duration as defined by the metronome. Therefore, positive values indicated longer movement durations than expected for the specific metronome pace; in contrast, negative values indicated shorter movement durations than expected for the specific metronome pace.

\subsection{Statistical analysis}

Because two of the seven talkers with ALS were severely affected by the disease, only the data of five participants with mild ALS and seven healthy controls were subjected to inferential statistical testing. The data of the two talkers with severe ALS were evaluated using descriptive statistics. In the lower lip fixed-target task, data of only four instead of five talkers with mild ALS were evaluated because one talker could not tolerate the bite block due to a hyperactive gag reflex.

To determine if there is a speed constraint in talkers with mild ALS, each participant's maximum peak speed that was observed across all four metronome paces during the jaw and lower lip fixed-target tasks was submitted to a one-tailed independent sample $t$-tests. A multivariate design was not completed due to the small number of participants and concerns about low statistical power.

To verify that participants refrained from using a displacement reductions during the fixed-target tasks and to rule out group differences in articulatory displacements, lower lip and jaw displacements of the slowest and fastest metronome pace were submitted to repeated measure ANOVAs with displacement as the repeated measure variable and groups as the between group variable. The slowest and fastest conditions were chosen because they elicited the full range of articulatory displacements from large to small, respectively.

Finally, to determine the durational accuracy of articulatory movements were in accordance with the metronome paces, the difference between the observed and expected movement duration was calculated for each participant during each task. The absolute error terms were submitted to an independent $t$-test for each metronome pace of both experimental tasks. 


\section{Results}

\subsection{Results of the fixed-target tasks}

\subsubsection{Peak speed}

To determine if talkers with ALS have limited lower lip and jaw speed capacities, each participant's maximum peak speed observed across all metronome paces were selected and a group mean was calculated for each group (Figure 4). One-tailed independent $t$-tests yielded significantly lower peak speeds of the lower lip in talkers with mild ALS $(M=134.15, \mathrm{SE}=8.18)$ than in controls $(M=194.54, \mathrm{SE}=21.40), p=.036$. Mean jaw peak speeds were not significantly different between the two groups; however, Figure 4 suggests that talkers with mild ALS tended to have slower jaw peak speeds $(M=101.36, \mathrm{SE}=15.90)$ than did healthy talkers $(M=136.32, \mathrm{SE}=16.01)$.

The left and right panels of Figure 5 display the mean peak speeds of each metronome pace for talkers with mild ALS and controls of the jaw and lower lip, respectively. Mean jaw peak speeds of controls tended to steadily increase as the metronome pace increased, whereas mean jaw peak speeds of talkers with mild ALS tended to increase from the slow to the fast metronome pace, but then decrease from the fast to the very fast metronome pace. The relative increase in speed tended to be greater in healthy controls than in talkers with mild ALS. Specifically, healthy talkers moved on average with lower jaw peak speeds during the slow metronome pace, but increased their jaw speed more rapidly with each metronome pace than did talkers with mild ALS. During the lower lip fixed-target task, movement speeds of healthy talkers were overall faster than those of the talkers with mild ALS. In addition, the increase in lower lip speed from the slow to the moderate metronome paces was smaller for the talkers with mild ALS than for the healthy controls.

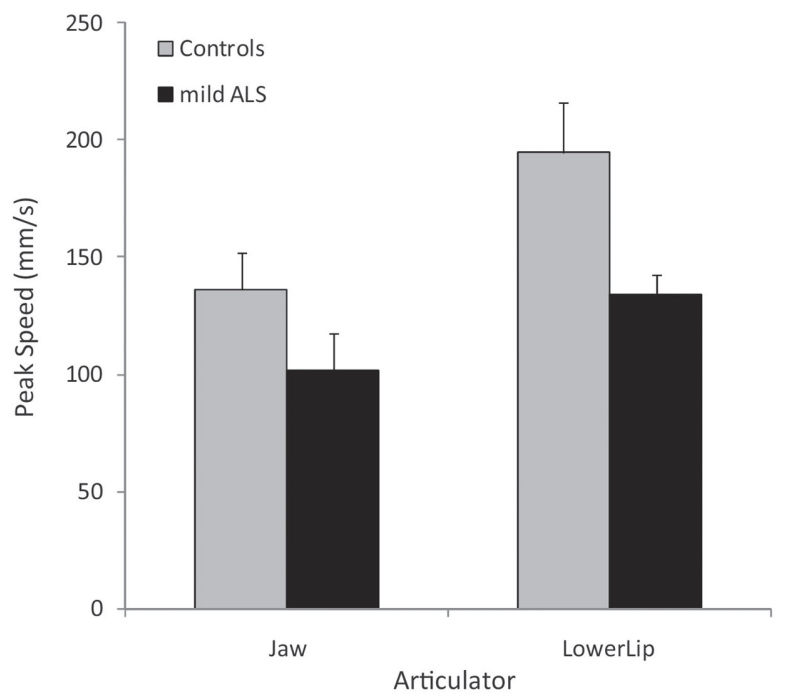

Figure 4. Mean peak speeds (+SE) of talkers with mild ALS and controls during the jaw fixed-target task (jaw) and lower lip fixed-target task (lower lip).

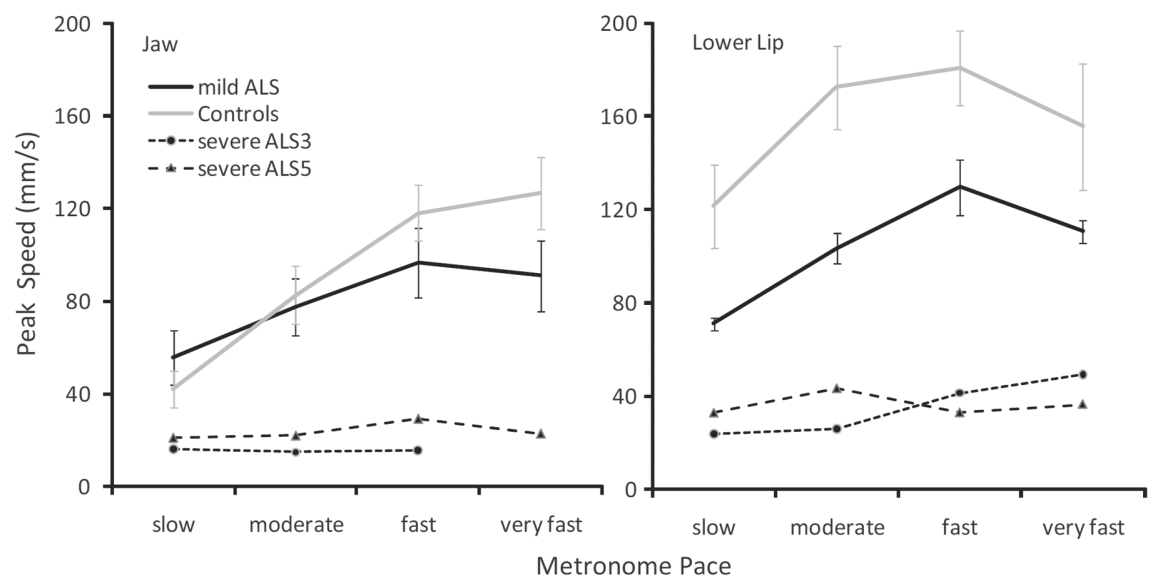

Figure 5. Mean peak speeds (+SE) of the jaw and lower lip for talkers with mild ALS and controls as well as individual data of two talkers with severe ALS. 


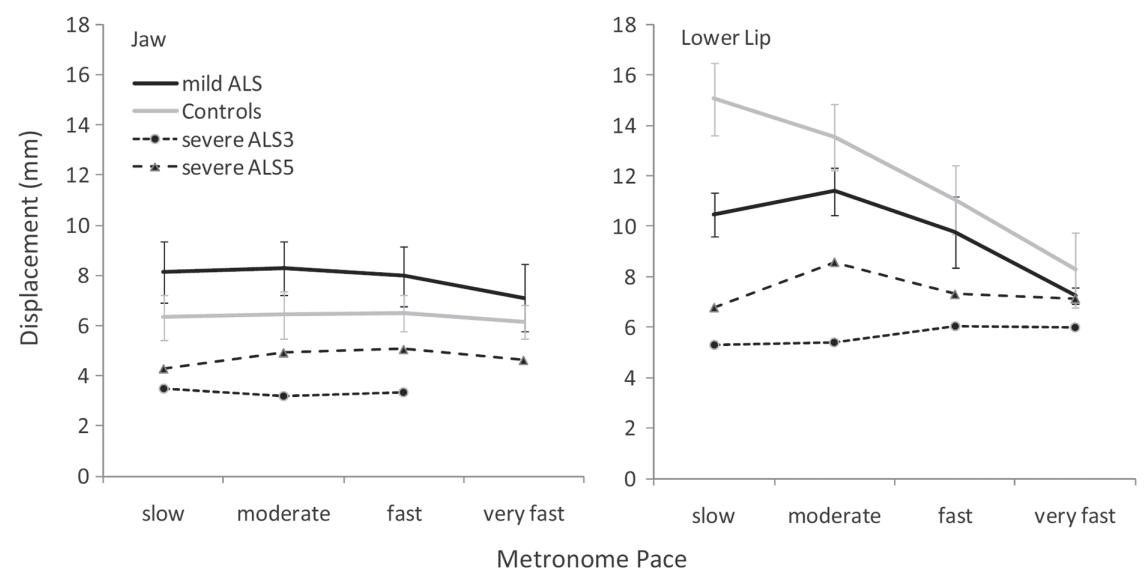

Figure 6. Mean maximum displacements (+SE) of the jaw and lower lip for talkers with mild ALS and controls as well as individual data of two talkers with severe ALS.

Jaw and lower lip peak speeds of the two talkers with severe ALS are displayed individually in Figure 5. For one talker with severe ALS (ALS3), data were missing for the very fast metronome pace during the jaw fixed-target task due to a recording error. In general, jaw and lower lip peak speeds were much slower in talkers with severe ALS than in talkers with mild ALS and healthy talkers. During the jaw fixed-target task, ALS3 (severe ALS) showed only minimal changes in jaw peak speed with increasing metronome pace. In contrast, ALS 5 (severe ALS) slightly increased jaw speed; however, to a much smaller extent than did talkers with mild ALS and controls. During the lower lip fixed-target task, lower lip peak speed slightly increased in both talkers with severe ALS. These changes were also much smaller than the observed speed increases in talkers with mild ALS and controls.

\subsubsection{Maximum displacement}

The left and right panels of Figure 6 display mean maximum displacements of jaw and lower lip, respectively. To evaluate the participants' ability to refrain from a "displacement reduction strategy" to increase oscillation rates, the movement displacements produced during the slowest and fastest metronome paces were submitted to a repeated measure ANOVA for each fixed-target task. In the jaw fixed-target task, the main effects for metronome pace and group, and the pace $\mathrm{x}$ group interaction were not significant. The left panel of Figure 6 , however, shows that the jaw displacements of talkers with mild ALS tended to be larger than those of healthy controls.

In the lower lip fixed-target task, the main effect for metronome pace was significant, $F(1,9)=25.852, p=.001$, with a significantly larger displacements during the slowest metronome pace $(M=12.8, \mathrm{SE}=1.02)$ than the fastest metronome pace $(M=7.8, \mathrm{SE}=1.00)$. Main effects for group and the group $\times$ metronome pace interaction were not statistically significant. The findings displayed in the right panel of Figure 6, however, suggest that the talkers with mild ALS tended to have smaller lower lip displacements than did healthy talkers during the slowest metronome pace. These group difference were not observed during the very fast pace.

Maximum jaw and lower lip displacements of the talkers with severe ALS are displayed individually in Figure 6. Jaw and lower lip displacements did not change with increasing metronome pace and were smaller in talkers with severe ALS than in talkers with mild ALS and controls during the slowest metronome pace. However, because talkers with mild ALS and controls decreased their lower lip displacements with increasing metronome pace, lower lip displacements were similar across all groups during the fastest metronome pace.

\subsubsection{Durational accuracy}

The right and left panels of Figure 7 display the observed mean duration of all participants and the expected target duration during the jaw and lower lip fixed-target tasks, respectively. The accuracy of movement durations for each metronome pace revealed significant differences between the two groups for the fast and very fast metronome paces during the jaw fixed-target task, $p=.004$ and $p<.001$, respectively. Specifically, healthy talkers were more accurate in their repetition rate than were talkers with mild ALS. The mean difference in durational accuracy between the talkers with mild ALS and healthy controls was slightly larger during the very fast metronome pace (Mean difference $=0.086 \mathrm{~s}$ ) than during the fast metronome pace (Mean difference $=0.085 \mathrm{~s}$ ). In addition, as depicted in Figure 7, talkers with mild ALS tended to match jaw oscillation rates more closely to the slow metronome pace than did healthy talkers. During the lower lip fixed-target task, healthy talkers tended to match their lower lip oscillation rates more closely to the very fast metronome pace than did talkers with mild ALS, $p=.06$. Mean differences between groups did increased from the fast metronome pace (Mean difference $=0.032$ ) to the fastest metronome pace (Mean difference $=0.036$ ). Further, during all metronome paces the talkers with severe ALS were less accurate in their lower lip and jaw movements than were talkers with mild ALS and healthy controls. 


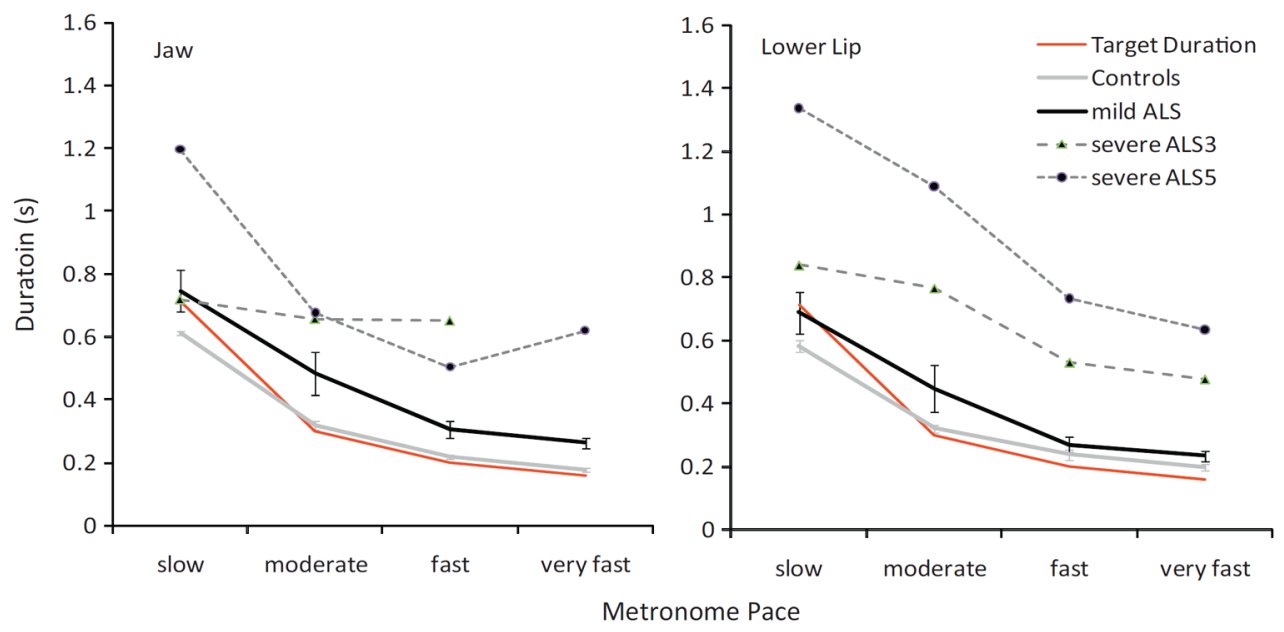

Figure 7. Mean movement durations (+SE) of the jaw and lower lip for talkers with mild ALS and controls as well as individual data of two talkers with severe ALS. The expected movement durations are also shown as a function of metronome pace.

\section{Discussion}

The goal of this study was to determine if talkers with mild ALS were limited in their lower lip and jaw speed capacities. Because previous studies have shown that healthy talkers may reduce articulatory displacement instead of increasing speed to achieve faster speech (Kuehn \& Moll, 1976), lip and jaw speed capacities were tested using a fixed-target paradigm. The fixed-target task required participants to focus their efforts on increasing lower lip and jaw speeds while matching a metronome pace that increasingly became faster. Results showed that lower lip speeds were significantly slower in talkers with mild ALS than in healthy talkers suggesting a speed constraint of the lower lip. Further, talkers with mild ALS showed trends towards lower jaw peak speeds in combination with larger jaw displacements relative to typical talkers suggesting a speed constraint of the jaw as well.

\subsection{Evaluation of the fixed-target task}

Although fixed-target tasks have been used to examine arm movements, these tasks are novel to the study of motor performance in the bulbar system. This study examined the feasibility of this approach in seven talkers with ALS and seven healthy controls. Results indicated that both, healthy talkers and talkers with ALS were able to complete the jaw fixed-target task by increasing jaw speed and maintaining jaw displacements. In contrast, the participants in both groups had difficulty moving their lower lips the full distance between the fixed targets at the fast metronome paces. Therefore, although the lower lip fixed-target task did not fully control for movement extent, it may have minimized the displacement reductions that talkers would typically implement under unconstrained conditions. The fixed-target task may have been less challenging to perform with the jaw than the lip because lip shape changed considerably during these tasks. Specifically, the lower lip was often stiffened and held in a retracted position against the teeth during the fast paces, which may explain why the lower lip displacements decreased with increasing metronome pace. The bite block, which was only used when testing lower lip speed capacity to prevent the jaw from moving, may have additionally resulted in somewhat awkward lower lip movements. Future work is required to determine if the lower lip fixed-target task minimizes the talker's tendency to use a lower lip displacement reductions compared to less constrained speech tasks.

\subsection{Speed capacities during the early stages of ALS}

The findings of this study suggest speed constraints of the lower lip and jaw during the early stages of the disease when speaking rate and intelligibility are only minimally affected. Group differences were statistically significant for the lower lip only; however, talkers with mild ALS tended to have lower jaw peak speeds than did healthy talkers. Based on the observation that jaw displacements were larger in the ALS group than in the healthy group across all metronome paces, jaw peak speeds should have been even greater for talkers with mild ALS than for healthy talkers because larger movements typically scale in proportion with greater speeds (Munhall et al., 1985; Nelson, 1983). This expected association between speed and displacement, however, was only observed during the slow metronome pace where both jaw displacements and jaw peak speeds of the talkers with mild ALS were greater than those of healthy talkers (Figure 5). With an increase in metronome pace, the discrepancy between the relatively large jaw displacements and relatively low jaw peak speeds became more evident in the ALS group. The lack of a linear as- 
sociation between movement speed and displacement of the jaw may reflect pathological changes in muscle stiffness in talkers with mild ALS (Kelso et al., 1986; Nelson, 1983) and a constrained ability to generate high jaw speeds.

The ALS group's limited ability to increase speed was anticipated based on previous findings that showed a slower rate of repeated contractions of jaw and lower lip muscles in persons with ALS relative to healthy controls (Langmore \& Lehman, 1994) and slowed lower lip speeds during vowel productions of monosyllabic words and diadochokinetic pseudo-speech tasks (Hirose et al., 1982a, 1982b; Yunusova et al., 2008). We, however, did not know whether speed constraints were already evident during the early stages of the disease because previous studies included talkers with obvious deterioration of motor performance. The preliminary results of this study suggest that movement speed is already affected when speech intelligibility and speaking rates are not or only minimally affected. Thus, movement speed decline may predate speaking rate and intelligibility decline in ALS. Larger scale studies are needed to examine changes in lower lip and jaw speed longitudinally to evaluate the potential of articulatory speed as a predictor of speech performance.

\subsection{Speed capacity during the late stages of ALS}

Prior to this study, the extent to which talkers with severe ALS could modulate their articulatory speed was unknown. A speed constraint was expected in talkers with severe ALS because motoneuron cell death reduces the rate and strength of muscular contractions. However, the collateral sprouting of axons from intact motor units to neighboring muscles is thought to effectively compensate for motor unit loss until approximately $80 \%$ of motoneurons have deteriorated (Brown, 1973; Wohlfart, 1957). Because only two talkers with severe ALS completed this study, our findings are very preliminary.

In general, talkers with severe ALS produced minimal variations in movement speed to increase their oscillation rates, whereas talkers with mild ALS were able to modulate speed. Despite the limited change in speed, talker ALS5 achieved notable durational changes. These durational changes were achieved by significant reductions of small submovements as the metronome pace increased. Such excessive submovements were not observed in the other talker with severe ALS or any other participants in the mild ALS group or healthy group. This finding suggests that the relations among speed, displacement, and movement duration may become more complex with increased speech severity.

Although talkers with mild ALS did not appear to have difficulty with the whispered syllable "fuh," both talkers with severe ALS displayed significant distortions during both fixed-target tasks. Specifically, syllable productions lacked sharp labiodentals airflow and were often produced with a slightly voiced fricative. The distortions may have multiple underlying factors including potentially decreased respiratory function, difficulty with vocal fold configuration to achieve whispering, as well as a decreased ability to move the lower lip independently of the jaw.

\subsection{Accuracy of metronome matching}

The duration data in Figure 7 suggest that the talkers with mild ALS were not as proficient as the healthy controls at matching the fast and very fast lower lip and jaw oscillation rates. Interestingly, however, talkers with mild ALS were also less accurate than controls while matching the moderate oscillation rates despite their speed capacity permitting accuracy during the moderate rate. Similarly, talkers with severe ALS displayed prolonged movement durations during the slow oscillation rates although these required rates were within their performance range. The reasons for the poor accuracy at slower oscillation rates are unclear, but these findings suggest that talkers with ALS have difficulties regulating articulatory speeds even when they are within their physiologic capacity.

During the slowest metronome pace, some participants of both groups performed oscillations with shorter than expected movement durations. These participants produced short syllables and paused between each production to match the slow metronome pace rather than adjusting their movement durations during syllable productions.

\subsection{Study limitations}

In this study, the distance to the fixed target was determined by each individual's natural displacement of the jaw or lower lip during the slowest metronome pace. We expected that the distance to the fixed target would be randomly distributed around a mean regardless of group membership. Results, however, showed that talkers with ALS tended to complete slow jaw oscillation rates with larger displacements and slower lower lip oscillation rates with smaller displacements than did healthy talkers. A constant distance across subjects was not used, however, because this approach would not take into consideration anatomical differences across participants and may have elicited unnatural movements in some talkers. We also noted that a pronounced double chin, a very thin lower lip, or a bulging mentalis muscle below the lower lip challenged the correct target placement to complete the fixed-target tasks.

\subsection{Conclusion}

This study showed that the fixed-target task is a feasible approach to test speed capacities in the bulbar system. The fixed-target task revealed that talkers with mild ALS may be constrained in their capacity to increase and modu- 
late lower lip and jaw speeds. These finding suggest that peak speed may be a sensitive measure to identify small changes in motor performance decline early, possibly even prior to changes in speaking rate and intelligibility. In the future, movement speed may be useful to predict the rate of disease progression within an individual. Longitudinal studies are warranted to better understand the potential of movement speed in the diagnosis and prognosis of ALS. Further, a fixed-target approach should be developed to assess tongue speed capacity because the tongue is the major articulator and predominantly affected in talkers with ALS.

Acknowledgments - This work was supported in part by research Grant numbers Ro1 DCoog89o and Ro1 DCo6463 from the National Institute on Deafness and Other Communication Disorders (NIDCD-NIH) awarded to the second author, by the Nebraska Speech-Language-Hearing Endowment Fund Dissertation Award awarded to the first author, and the Barkley Trust. We would like to thank Cynthia Didion, Cara Ullman, Ignatius Nip, Lindsey Fairchild, Paige Leising, and Jenna Mroczek for their assistance with data collection and management.

\section{Appendix A. Continuing education}

(1) To produce fast speech talkers often decrease their articulatory displacements.True/False

(2) A fixed-target task experimentally controls
(a) Movement direction
(b) Movement speed
(c) Movement distance
(d) Rate of movement

(3) The syllable "buh" is not suited for a fixed-target task because

(a) The upper lip moves closer to the lower lip to achieve bilabial closure

(b) Jaw movements are only minimal during these syllable productions

(c) Lower lip movements are not independent of the jaw

(4) The assessment of orofacial speed capacities may be useful in talkers with ALS

(a) To predict the rate of speech decline within an individual

(b) To provide objective outcome measures in therapeutic drug trials

(c) To expedite the diagnosis

(d) All of the above

(5) Findings of this study provided evidence that talkers with mild ALS had no lower lip and jaw speed constraints. True/False

\section{References}

Ball, L. J., Beukelman, D. R., \& Pattee, G. L. (2002). Timing of speech deterioration in people with amyotrophic lateral sclerosis. Journal of Medical Speech-Language Pathology, 10(4), 231-235.

Beukelman, D., Fager, S., \& Nordness, A. (2011). Communication support for people with ALS. Neurology Research International, 2011, 6; doi:10.1155/2011/714693 (Article ID 714693).

Brooks, B. R. (1996). Natural history of ALS: Symptoms, strength, pulmonary function, and disability. Neurology, 47(S 2), S71-S82.

Brown, W. F. (1973). Functional compensation of human motor units in health and disease. Journal of the Neurological Sciences, 20, 199-209.

Darley, F. L., Aronson, A. E., \& Brown, J. R. (1969a). Clusters of deviant speech dimensions in the dysarthrias. Journal of Speech and Hearing Research, 12(3), 462-496.

Darley, F. L., Aronson, A. E., \& Brown, J. R. (1969b). Differential diagnostic patterns of dysarthria. Journal of Speech and Hearing Research, 12(3), 246-269.

DePaul, R., Abbs, J. H., Caligiuri, M. P., Gracco, V. L., \& Brooks, B. R. (1988). Differential involvement of hypoglossal, trigeminal and facial motoneurons in ALS. Neurology, 38(2), 281-283.

DePaul, R., \& Brooks, B. R. (1993). Multiple orofacial indices in amyotrophic lateral sclerosis. Journal of Speech and Hearing Research, 36, 1158-1167. 
Green, J. R., Wilson, E. M., Wang, Y., \& Moore, C. A. (2007). Estimating mandibular motion based on chin surface targets during speech. Journal of Speech, Language, and Hearing Research, 50, 928-939.

Hertrich, I., \& Ackermann, H. (200o). Lip-jaw and tongue-jaw coordination during rate-controlled syllable repetitions. Journal of the Acoustical Society of America, 107(4), 2236-2247.

Hirose, H., Kiritani, S., \& Sawashima, M. (1982a). Patterns of dysarthric movement in patients with amyotrophic lateral sclerosis and pseudobulbar palsy. Folia Phoniatrica et Logopaedica, 34, 106-112.

Hirose, H., Kiritani, S., \& Sawashima, M. (1982b). Velocity of articulatory movements in normal and dysarthric subject. Folia Phoniatrica et Logopaedica, 34, 210-215.

Kelly, V. E., \& Bastian, A. J. (2005). Antiparkinson medications improve agonist activation but not antagonist inhibition during sequential reaching movements. Movement Disorders, 20(6), 694-704.

Kelso, J. A. S., Saltzman, E. L., \& Tuller, B. (1986). The dynamical perspective on speech production: Data and theory. Journal of Phonetics, 14, 29-59.

Kuehn, D. P., \& Moll, K. L. (1976). A cineradiographic study of VC and CV articulatory velocities. Journal of Phonetics, 4, 303-320.

Langmore, S. E., \& Lehman, M. E. (1994). Physiologic deficits in the orofacial system underlying dysarthria in amyotrophic lateral sclerosis. Journal of Speech and Hearing Research, 37, 28-37.

Lindblom, B. (1990). Explaining phonetic variation: A sketch of the H\&H theory. In W. J. Hardcastle \& A. Marchal (Eds.), Speech production and speech modelling (pp. 404-440). Dordrecht, the Netherlands: Kluwer Academic Publishers.

Mefferd, A. S., \& Green, J. R. (2010). Articulatory-to-acoustic relations in response to speaking rate and loudness manipulation. Journal of Speech, Language, and Hearing Research, Vol.53, 1206-1219.

Milner, T. E., \& Ijaz, M. (1990). The effect of accuracy constraints on three-dimensional movement kinematics. Neuroscience, 35, 365-374.

Munhall, K. G., Ostry, D. J., \& Parush, A. (1985). Characteristics of velocity profiles of speech movements. Journal of Experimental Psychology: Human Perception and Performance, 11, 457-474.

Nelson, W. L. (1983). Physical principals for economies of skilled movements. Biological Cybernetics, 46(2), $135-147$.

Perkell, J., Matthies, M., Lane, H., Guenther, F., Wilhelms-Tricarico, R., Wozniak, J., et al. (1997). Speech motor control: Acoustic goals, saturation effects, auditory feedback and internal models. Speech Communication, 22, 227-250.

Rand, M. K., Squire, L. M., \& Stelmach, G. E. (2006). Effect of speed manipulation on the control of aperture closure during reach-to-grasp movements. Journal of Experimental Brain Research, 174(1), 74-85.

Wohlfart, G. (1957). Collateral regeneration from residual motor neuron fibers in amyotrophic lateral sclerosis. Neurology, 7, 124-134.

Yorkston, K., Beukelman, D. R., Hakel, M., \& Dorsey, M. (2007). Speech intelligibility test for windows [computer software]. Lincoln, NE: Institute for Rehabilitation Science and Engineering at Madonna Rehabilitation Hospital.

Yorkston, K., Strand, E., Miller, R., Hillel, A., \& Smith, K. (1993). Speech deterioration in amyotrophic lateral sclerosis: Implications for the timing of intervention. Journal of Medical Speech-Language Pathology, 1, 35-46.

Yunusova, Y., Green, J. R., Lindstrom, M. J., Ball, L. J., Pattee, G. L., \& Zinman, L. (2010). Kinematics of disease progression in bulbar ALS. Communication Disorders, 43(1), 6-20.

Yunusova, Y., Weismer, G., Westbury, J. R., \& Lindstrom, M. J. (2008). Articulatory movements during vowels in speakers with dysarthria and healthy controls. Journal of Speech, Language, and Hearing Research, 51, 596-611. 